Orthopädie

René Baumgartner, Bernhard Greitemann

\section{Grundkurs Technische Orthopädie}

Stuttgart: Georg Thieme Verlag; 2007

2., überarbeitete Auflage, kartoniert

260 Seiten, 625 Abbildungen, 12 Tabellen

Fr. 115.-/€ 69.95

ISBN 978-3-13-125072-8

\section{Grundkurs \\ Technische Orthopädie}

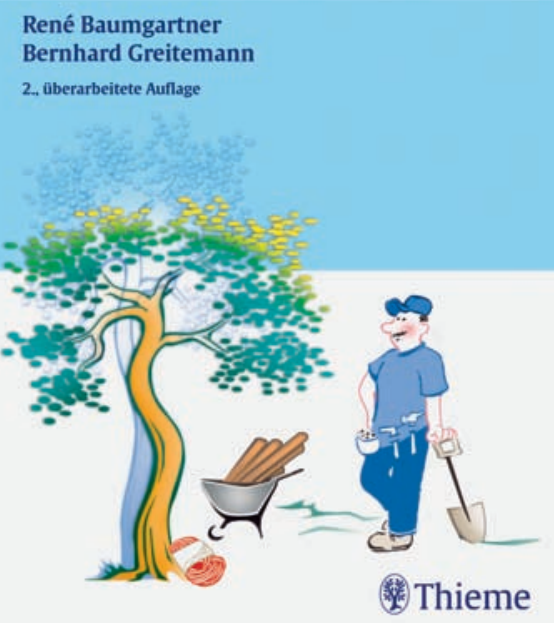

Der Schöpfer der Orthopädie, Nicolas Andry, hat 1741 «Das krumme Bäumchen, gerichtet durch Pfahl und Seil» zum Symbol des Faches gemacht. Das vorliegende Buch macht deutlich, was sich alles hinter «Pfahl und Seil» verbirgt. Die Technische Orthopädie, obwohl sie gegenüber der operativen Orthopädie heutzutage weit ins Hintertreffen geraten ist, hat sich seit ihren Anfängen enorm weiterentwickelt. Durch neue biomechanische Erkenntnisse und neue Technologien ist die technische Orthopädie effizienter und interessanter geworden. Die Möglichkeiten und die Auswahl sind vielfältig. Die Technische Orthopädie ist die Lehre der Orthetik, Prothetik, orthopädietechnischen Hilfsmittel, Schuhtechnik und Amputationstechnik. Die Erkenntnis, dass die Orthopädietechnik ein wichtiges Standbein der Orthopädie darstellt, beginnt sich in deutschsprachigen Regionen endlich wieder durchzusetzen, was sich auch im Zusammenrücken der Orthopädie und Unfallchirurgie ganz aktuell zeigt. Gerade in der Amputationschirurgie wird deutlich, dass die Chirurgie allein nicht zum endgültigen erwünschten Ergebnis führen kann. Sie ist untrennbar mit der Prothesenversorgung verbunden. Erfahrungsaustausch und Zusammenarbeit der beiden Disziplinen sind notwendig.

Die Autoren geben mit der systematischen Beschreibung und Abbildung aller Prothesenund Orthesengruppen zu den verschiedenen Körperregionen eine klare Übersicht für die Praxis. Sie haben das bewährte Schema aus der ersten Auflage beibehalten, das Prinzip, Indikationen, Ausführung und Verordnung sowie Abnahmepunkte beinhaltet. Ausserdem geben die Autoren zu jedem Hilfsmittel eine subjektive Beurteilung, was den Praxiswert des Buches erhöht, da man direkt aus ihren reichen klinischen Erfahrungen schöpfen kann. Ebenso wurde auf gesetzliche Grundlagen aufmerksam gemacht und eine Unterweisung in Fragen der Kostenübernahme gegeben.

Das zehnte Kapitel beinhaltet ein Diagnoseregister, dessen Sinn es ist, bestimmte orthopädische Krankheitsbilder und deren orthopädietechnische Versorgungsmöglichkeiten rasch aufzufinden. Es wird zunächst das Krankheitsbild in Stichworten vorgestellt und auf die in den vorangegangenen Kapiteln beschriebenen orthopädietechnischen Versorgungsmöglichkeiten verwiesen.

Ein weiteres Plus mit praktischem Wert sind die Formulierungshilfen, die die Rezeptierung erleichtern, sowie die zahlreichen Abbildungen, die auch zur instruktiven Patientenaufklärung genutzt werden können. Das Buch sollte nicht nur Orthopädietechniker interessieren, sondern vor allem auch orthopädischchirurgisch tätige Ärzte, die damit mit wenig Aufwand die wichtigsten Grundkenntnisse erwerben können. Es handelt sich um ein ausgezeichnetes praxisorientiertes Nachschlagewerk mit hohem Informationsgehalt.

Sandra Krüger, Neuenburg 